\title{
The IgE-diagnostic impact of a recombinant serine protease inhibitor from Hevea brasiliensis in latex-allergic health care workers
}

\author{
Hans-Peter Rihs ${ }^{\text {* }}$, Ingrid Sander ${ }^{2}$, Heike Heimann ${ }^{1}$, Ursula Meurer ${ }^{2}$, Thomas Brüning ${ }^{3}$, Monika Raulf-Heimsoth² \\ From 5th International Symposium on Molecular Allergology (ISMA 2013) \\ Vienna, Austria. 6-7 December 2013
}

\section{Background}

Serine protease inhibitors (SPI) comprising 60-90 amino acid residues, are frequent in plants, and belong to the protein family PR-6. It has recently been shown that a SPI-variant of wheat acts as an important allergen in baker's asthma but not in persons suffering from wheatinduced food allergy. The aim of this study was to elucidate the role of a recombinant SPI-variant of Hevea brasiliensis for occupational latex allergy.

\section{Methods}

For this purpose a cDNA from Hevea brasiliensis was used to amplify the SPI-specific sequence. After identification by sequencing and transfer into the pMAL-system, the sequence was expressed as maltose-binding protein (MBP)-SPI hybrid in E.coli. The soluble purified fusion protein was biotinylated and coupled to StreptavidinImmunoCAP. Sera of 21 health care workers (HCW) with allergic symptoms to natural rubber latex (NRL) were tested.

\section{Results}

Specific IgE (sIgE)-values $>0.35 \mathrm{kUA} / \mathrm{L}$ were regarded as positive. Seven sera (33\%) displayed SPI-sIgE (range: 0.56-13.60 kUA/L). Out of these seven sera all displayed sIgE to rHev b 6.01 (range: $1.81-66.80 \mathrm{kUA} / \mathrm{L}$ ) and with one exception also sIgE to $\mathrm{rHev}$ b $5(1.86-49.6 \mathrm{kUA} / \mathrm{L})$. Furthermore, positive sIgE to nHev b $2(n=6 / 7)$ and rHev b $7(n=4 / 7)$ were most frequently. No sIgE to MBP was detectable.

\section{Conclusions}

The results show that this SPI-variant is a further latex allergen, which is available now for extended tests, for instance on microarray platforms. The proposed name Hev b 15 is currently under review of the WHO/IUIS allergen nomenclature subcommittee. Especially in patients showing discrepancies between the sum of sIgE-values to single NRL-allergens and the IgE-value to NRL k82, Hev b 15 may be one candidate filling the gap.

\section{Authors' details}

1 Institute for Prevention and Occupational Medicine of the German Social Accident Insurance (IPA), Molecular Genetics, Bochum, Germany. ${ }^{2}$ Institute for Prevention and Occupational Medicine of the German Social Accident Insurance (IPA), Allergology/Immunology, Bochum, Germany. ${ }^{3}$ Institute for Prevention and Occupational Medicine of the German Social Accident Insurance (IPA), Head of the Institute, Bochum, Germany.

Published: 17 March 2014

doi:10.1186/2045-7022-4-S2-P56

Cite this article as: Rihs et al:: The IgE-diagnostic impact of a recombinant serine protease inhibitor from Hevea brasiliensis in latexallergic health care workers. Clinical and Translational Allergy 2014 4(Suppl 2):P56. 